\title{
Determination of 31 pesticide residues in wolfberry by LC-MS/MS and dietary risk assessment of wolfberry consumption
}

\author{
Lijie XING ${ }^{1,2,3}$, Yuan WANG ${ }^{1,2,3,4 \star} \mathbb{D}$, Ruifeng LUO $^{1,2,3}$, Xianyi LI ${ }^{1,2,3}$, Liangjun ZOU ${ }^{1,2,3}$
}

\begin{abstract}
A modified QuEChERS method coupled with LC-MS/MS was developed and validated to detect 31 pesticides in wolfberry. The conditions for extraction solvent and QuEChERS purification were optimized. The validated method was applied to analyse pesticides in 200 wolfberry samples. The risk from chronic and acute dietary intake of the detected pesticide residues was assessed by the acceptable daily intake $(A D I)$ and acute reference dose $(A R f D)$, respectively, and the cumulative intake risk posed by the detected residues was assessed by the hazard index $(H I)$. The results showed that 23 pesticides were detected in all wolfberry samples. The risk from chronic dietary intake was between $0.0001 \%$ and $1.6067 \%$, and the risk from acute dietary intake was between $0.0010 \%$ and $0.4999 \%$, which were all far below $100 \%$. The $H I$ was 0.02569 for chronic dietary intake and 0.015164 for acute dietary intake, which were both far below 1 . The results indicated that the pesticide residues in wolfberry would not cause potential risk to human health. This work not only enhances our understanding of the potential exposure risks of pesticide residues in wolfberry, but also provides an effective method for the risk assessment of pesticide residues in other agricultural products.
\end{abstract}

Keywords: wolfberry; pesticide residues; modified QuEChERS; LC-MS/MS; risk assessment.

Practical Application: In China, wolfberry is considered as medicinal and edible plants, which are popular among consumers for their ability to improve human body function. However, due to the long planting history, the occurrence of diseases and insect pests is more and more serious, and the use of pesticides to control diseases and insect pests brings risk to the consumption of wolfberry. In this study, an LC-MS/MS method was established for the determination of 31 pesticide residues in wolfberry, and the pesticide residues of wolfberry in northwest China were determined, and dietary risk assessment was carried out. This has important significance to the safe consumption of wolfberry in the future. At the same time, it also provides a reference for the use of pesticides in the planting process of this plant.

\section{Introduction}

Wolfberry (Lycium barbarum L.) is a perennial deciduous shrub with ellipsoid orange-red berries (Zhao et al., 2015). It is a Solanaceae plant mainly found in Northwest China, including Xinjiang Province, Qinghai Province, Ningxia Province, Gansu Province and other provinces. Wolfberry has a long history in China and has been used for medicine and functional food, and it is listed in the Traditional Chinese Pharmacopeia (TCP) (Amagase \& Farnsworth, 2011; Lu et al., 2014). To a certain extent, wolfberry has been one of the most important products exported from these provinces and has provided a significant contribution to the local economy (Ali et al., 2019).Wolfberry dried fruit contains many nutrients, such as polysaccharide, phenolic acid, carotene, betaine and flavonoids (Donno et al., 2016; Qian, 2004; Wang et al., 2010), which possesses many advantages, such as antioxidation, antiradiation, anticancer, anti-ageing characteristics, enhancement of haemopoiesis, brightening of the eyes, etc., which has increased its popularity among consumers (Chiu et al., 2010; Gan et al., 2004; Luo et al.,
2004; Zhou et al., 2017). Therefore, wolfberry, as a kind of food with great health benefits, is not only loved by consumers in China and Southeast Asia but also consumed in European and American markets, which is expanding year by year (Potterat, 2010). However, due to its high sugar content, wolfberry is vulnerable to aphids, psyllids, gall mites and other pests and also susceptible to root rot, anthracnose and powdery mildew (Chawla et al., 2017).

In recent years, pesticide residues in wolfberry have become the focus of consumers' attention. To ensure the production and quality of wolfberry, insecticides, acaricides and fungicides are used in the process of wolfberry cultivation. Thus, a large amount of pesticide residue is contained in wolfberry, which represents hidden safety risks to consumers' human health (Huang et al., 2012). Therefore, carrying out risk assessments of pesticide residues in wolfberry is an important means to control the quality and safety of wolfberry and has important significance for protecting consumer health. 
The QuEChERS (quick, easy, cheap, effective, rugged and safe) method has been broadly applied for the analysis of pesticide multi-residues in fruits and vegetables because of its simplicity, low cost, speed and broad applicability to a wide range of analytes (Anastassiades et al., 2003). At present, there are few methods for simultaneous determination of pesticide residues in wolfberry by LC-MS/MS (Chen et al., 2019). In particular, there are a few studies on the risk assessment of pesticide residues in wolfberry. Liu et al. (2015) performed risk evaluations of the main pests and integrated management of Chinese Wolfberry. Li et al. (2020) performed a dissipation study and dietary risk assessment of dinotefuran, DN, and UF in wolfberry. Qin et al. (2020) determined pesticide residues of pyrethrins in Lyciumbarbarum (goji) by GC-MS/MS and analysed a dietary risk assessment of Chinese goji consumption. Fu et al. (2017) studied eight pesticides in Lyciumbarbarum by LC-MS/MS and analysed a dietary risk assessment. The above work mainly included the risk assessment of major diseases in wolfberry and risk assessment of a small part of pesticides, but there were no reports on the risk assessment of pesticide residues, such as triazole, carbendazine and amidine, which were often used in the cultivation process of wolfberry.

The main purpose of this work is to establish a modified QuEChERS method coupled with LC-MS/MS for the determination of 31 pesticide residues in wolfberry, and analyse the residual levels of 31 pesticides in these wolfberry samples from Northwest China. In addition, we also carried out chronic dietary intake risk assessment, acute dietary intake risk assessment and cumulative risk assessment of pesticides present in the wolfberry samples to assess the potential risks caused by human exposure to these pesticides. This study would establish a method for pesticide multi-residues analysis in wolfberry, and also support the risk managers for developing monitoring programs and further guide the rational use of pesticides.

\section{Materials and methods}

\subsection{Chemicals, reagents and standards}

Deionized water $(>18.2 \mathrm{M} \Omega$ ) was prepared with a Milli-Q water purification system (Millipore Corp., USA). HPLC-grade formic acid, acetone, $\mathrm{n}$-hexane and acetonitrile were purchased from Merck Co. (Darmstadt, Germany). Primary secondary amine (PSA), octadecylsilane (C18) and graphitized carbon black (GCB) for use as absorbents were all provided by CNW Technologies GmbH (Düsseldorf, Germany). Analytical reagentgrade sodium chloride $(\mathrm{NaCl})$ and anhydrous magnesium sulfate $\left(\mathrm{MgSO}_{4}\right)$ were supplied by Tianjin Kemiou Chemical Reagent Co., Ltd. (Tianjin, China).

Standards of 31 pesticides were purchased from Dr.Ehrenstorfer (Augsburg, Germany), all with purity $>98 \%$. Individual pesticide stock solutions $(1000 \mathrm{mg} / \mathrm{L})$ were prepared in acetonitrile for LC-MS/MS analysis. All individual standard stock solutions were kept in a refrigerator at $-20^{\circ} \mathrm{C}$. For optimization and calibration, working solutions were prepared daily by appropriate dilution of the stock standard solutions, which were kept at $-4{ }^{\circ} \mathrm{C}$. For optimization of the ion source parameters for LCMS/MS, individual standard solutions of each pesticide were prepared at $10 \mu \mathrm{g} / \mathrm{mL}$ in acetonitrile. For the calibration studies, working standard solution mixtures were prepared at different concentrations in $\mathrm{ACN}: \mathrm{H}_{2} \mathrm{O}(10: 90, v / v$, containing $0.1 \% \mathrm{FA})$ for LC-MS/MS.

\subsection{Samples}

A total of 200 samples of dried wolfberry fruit were collected from the main cultivation areas in Xinjiang Province and Qinghai Province in Northwest China: 100 samples from Xinjiang and 100 samples from Qinghai. The samples were stored in sealed bags, refrigerated, transported to the laboratory, and stored at $-20^{\circ} \mathrm{C}$ until preparation and analysis.

\subsection{Sample preparation}

Wolfberry $(2.00 \mathrm{~g})$ was accurately weighed and transferred into a $50-\mathrm{mL}$ plastic centrifuge tube. Then, $5 \mathrm{~mL}$ of water and $10 \mathrm{~mL}$ of acetonitrile were added sequentially to the tube. The mixture was vortexed for $30 \mathrm{~s}$ (Vortex Genie 2 vortex mixer, Scientific Industries Inc., USA) and extracted by ultrasonication for 15 min (KQ-1000 ultrasonic cleaner, Shanghai Baidian Instrument Factory, China). Then, $1.0 \mathrm{~g}$ of $\mathrm{NaCl}$ was added to the tube, and the mixture was vortexed for $1 \mathrm{~min}$ and then centrifuged for $5 \mathrm{~min}$ at $2500 \mathrm{~g}$ (3-30 K high-speed centrifuge, Sigma Laborzentrifugen $\mathrm{GmbH}$, Germany). Five millilitres of the extract was transferred into a $15-\mathrm{mL}$ plastic high-speed centrifuge tube preloaded with $400 \mathrm{mg} \mathrm{C} 18,400 \mathrm{mg}$ PSA, $45 \mathrm{mg}$ GCB and $1200 \mathrm{mg} \mathrm{MgSO}_{4}$. The above mixture was vortexed for $1 \mathrm{~min}$ and centrifuged for $3 \mathrm{~min}$ at $10000 \mathrm{~g}$. Then, $1.0 \mathrm{~mL}$ of supernatant each was transferred into two clean $10-\mathrm{mL}$ glass colorimetric tubes and dried under flowing nitrogen gas in a $40{ }^{\circ} \mathrm{C}$ water bath (nitrogen evaporator, Organomation Co., USA). Sample was redissolved in $1.0 \mathrm{~mL}$ of acetonitrile- $0.1 \% \mathrm{FA}$ water (10:90, $v / v)$, filtered through a $0.22-\mu \mathrm{m}$ organic filter membrane, and analysed by LC-MS/MS. If the pesticide concentrations in the wolfberry samples exceeded the linear range, the solutions were appropriately diluted with the corresponding solvent.

\subsection{LC-MS/MS analysis}

A UPLC-MS/MS system consisting of a Waters ACQUITY UPLC unit and a Xevo TQ-S mass spectrometer (Waters Co., USA) was used for the separation and quantitation of 25 pesticides. Chromatographic separation was performed on a BEH C18 analytical column $(100 \mathrm{~mm} \times 2.1 \mathrm{~mm}, 1.7 \mu \mathrm{m}$, Waters Co., USA), and the column temperature was maintained at $30^{\circ} \mathrm{C}$. The flow rate was maintained at $0.3 \mathrm{~mL} / \mathrm{min}$, and the injection volume was $5 \mu \mathrm{L}$. The mobile phase consisted of water (containing $0.1 \% \mathrm{FA}, v / v$ ) and acetonitrile. The following linear gradient elution procedure was adopted for separation of the 31 pesticides: $0.0-2.0 \mathrm{~min}, 10-50 \%$ acetonitrile; $2.0-2.1 \mathrm{~min}, 50$ $90 \%$ acetonitrile; $2.1-4.0 \mathrm{~min}, 90 \%$ acetonitrile; and $4.0-5.0 \mathrm{~min}$, 90-10\% acetonitrile.

The mass spectrometer contained a Z-spray electron spray ionization (ESI) source. The ion source parameters were as follows: positive mode, capillary voltage of $3.20 \mathrm{kV}$, source temperature of $150^{\circ} \mathrm{C}$, desolvation temperature of $400^{\circ} \mathrm{C}$, desolvation gas flow rate of $800 \mathrm{~L} / \mathrm{h}$, cone gas flow rate of $50 \mathrm{~L} / \mathrm{h}$ and collision gas (Ar) 
flow rate of $0.20 \mathrm{~mL} / \mathrm{min}$. The cone voltage $(\mathrm{CV})$, parent ions, collision energy (CE) and fragment ions were optimized for each pesticide using MassLynxIntelliStar software. The 31 pesticides were analysed in multiple reaction monitoring (MRM) mode. Data acquisition and processing were accomplished using MassLynx TM 4.1 software.

\subsection{Chronic risk assessment}

The risk posed by the chronic dietary intake of each pesticide in wolfberry was calculated as the acceptable daily intake percentage $(\% A D I)$. The smaller the \%ADI value is, the lower the risk. $\% A D I \leq 100 \%$ indicates acceptable risk; and a value above $100 \%$ indicates unacceptable risk. Formula 1 presents the $\% A D I$ calculation.

$$
A D I \%=\frac{S T M R \times P}{A D I \times \mathrm{bw}} \times 100
$$

Where STMR $(\mathrm{mg} / \mathrm{kg})$ is the supervised trial median residue, $A D I(\mathrm{mg} / \mathrm{kg})$ is the acceptable daily intake, $P(\mathrm{~kg})$ is the daily consumption of wolfberry for residents calculated based on the dry fruit consumption of wolfberry recommended by the Chinese Pharmacopoeia of $0.012 \mathrm{~kg}$ per day for adults, and $b w(\mathrm{~kg})$ is the human body weight (assumed to be $60 \mathrm{~kg}$ for an adult).

\subsection{Acute risk assessment}

The risk posed by acute dietary intake of each pesticide in wolfberry was calculated as the acute reference dose percentage $(\% A R f D)$. The smaller the \%ARfD value is the lower the risk. $\% A R f D \leq 100 \%$ indicates acceptable risk; and a value above $100 \%$ indicates unacceptable risk. \%ARfD and the safety margin $(S M)$ of each pesticide were calculated by Formulas 2 and 3, respectively.

$\% A R f D=\frac{H R \times P}{A R f D \times b w} \times 100$

$S M=\frac{A R f D \times b w}{P}$

Where HR $(\mathrm{mg} / \mathrm{kg})$ is the highest residue concentration and $A R f D(\mathrm{mg} / \mathrm{kg})$ is the acute reference dose.

\subsection{Cumulative risk assessment}

The cumulative risk assessment of each pesticide in wolfberry was carried out on the basis of the hazard index $(H I)$. The $H I$ is the sum of the hazard quotient (HQ) of each chemical, as shown in Formula 6. HQ is the ratio of the exposure (EXP) to reference $(R V)$ values. The calculation of acute dietary exposure (EXP $a)$ and chronic dietary exposure $(E X P c)$ is shown in Formulas 4 and 5, respectively. When $H I$ is less than 1 , the cumulative exposure risk is acceptable; otherwise, the risk is unacceptable.

$$
\begin{aligned}
& E X P \mathrm{a}=\frac{H R \times P}{b w} \\
& E X P c=\frac{S T M R \times P}{b w}
\end{aligned}
$$

$H I=\sum_{i-1}^{n} H Q i=\frac{E X P}{R V}$

The $A D I$ is the reference value for chronic toxicity, and $A R f D$ is the reference value for acute toxicity. Referring to the maximum daily consumption value of $97.5 \%$ proposed in the global environmental monitoring system/food pollution monitoring and assessment plan (GEMS/Food), the value of $P$ in wolfberry in this study was $10.7 \mathrm{~g} / \mathrm{kg}$.

\section{Results and discussion}

\subsection{Optimization of the extraction solvent}

The target pesticides covered by this study mainly include 31 pesticides, including organophosphate, benzimidazole, carbamate, triazole, avermectin and methylcarbamate. To minimize the interference of the co-extracted materials and improve the extraction efficiency of the 31 pesticides, the extraction solvent was investigated. In this study, acetonitrile and ethyl acetate as the extraction solvents were compared. The results indicated that the recoveries of all the pesticides extracted with in acetonitrile were satisfactory and ranged between $80.4 \%$ and $110.4 \%$, and the recoveries of all the pesticides extracted with in ethyl acetate were ranged between $68.2 \%$ and $114.6 \%$. As shown in Figure 1, the extraction efficiencies for the 31 pesticides with acetonitrile as the extraction solvent were better than those obtained with ethyl acetate. acetonitrile extraction is commonly and widely applied in QuEChERS method since it leads to less interference, such as lipophilic compounds, waxes, lipids, and pigments, and other extract solvents, such as acetone and ethyl acetate (Anastassiades et al., 2003).

\subsection{Optimization of the QuEChERS purification}

After the wolfberry samples were extracted in acetonitrile, the extraction solution was not qualified for LC-MS/MS analysis due to a large amount of interference and need for purification (Oshita \& Jardim, 2014). QuEChERS purification techniques have been widely applied in the agricultural products and food detection fields. Some sorbents, such as C18, PSA and GCB, are commonly employed in QuEChERS procedures. The purification efficiencies of different absorbents were evaluated in this study. Stock solutions were diluted with blank wolfberry extract to prepare a mixed standard working solution at a certain concentration. Two millilitres of the upper acetonitrile solution was accurately transferred into a $10 \mathrm{~mL}$ centrifuge tube pre-loaded with mixtures in different proportions of C18 adsorbent, PSA adsorbent and GCB adsorbent (nine levels). Then, the extraction solutions were vortexed and centrifuged. Next, $1 \mathrm{~mL}$ of the supernatant was transferred to a glass centrifuge tube and dried under flowing nitrogen in a $40{ }^{\circ} \mathrm{C}$ water-bath. Subsequently, the residue was redissolved $1.0 \mathrm{~mL}$ of $10 \%$ acetonitrile- $0.1 \% \mathrm{FA}$ in water $(v / v)$ and filtered through a $0.22-\mu \mathrm{m}$ organic filter membrane. Finally, the supernatant was determined by LC-MS/MS, and the recovery was calculated. The results are shown in Table 1 . When $400 \mathrm{mg}$ C18, $400 \mathrm{mg}$ PSA, and $45 \mathrm{mg}$ GCB are added, the purification efficiencies are best for the 31 pesticides. The purification efficiencies with the $400 \mathrm{mg}$ adsorbents (C18 and PSA) were higher than 


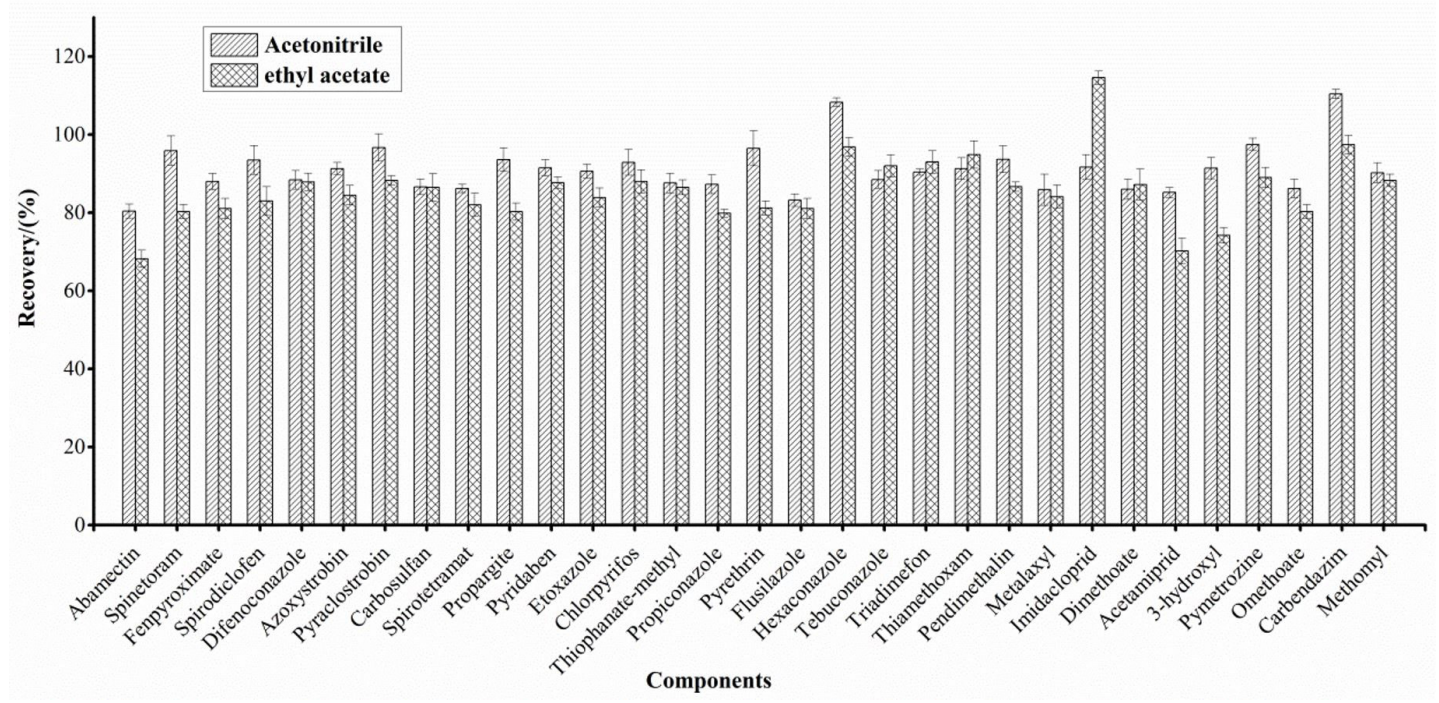

Figure 1. Comparison of extraction efficiency of different extraction solvents.

Table 1. Recoveries of 31 pesticides purified with different absorbent types and dosages (Spiked $20 \mu \mathrm{g} / \mathrm{kg}, \mathrm{n}=3$ ).

\begin{tabular}{|c|c|c|c|c|c|c|c|c|c|}
\hline \multirow{2}{*}{ Components } & \multicolumn{9}{|c|}{ Recoveries (mean \pm SD, \%) } \\
\hline & A & $\mathrm{B}$ & $\mathrm{C}$ & $\mathrm{D}$ & $\mathrm{E}$ & $\mathrm{F}$ & G & $\mathrm{H}$ & $\mathrm{I}$ \\
\hline Abamectin & $77.6 \pm 0.8$ & $79.7 \pm 1.3$ & $74.1 \pm 1.7$ & $81.3 \pm 1.2$ & $83.5 \pm 1.5$ & $82.2 \pm 1.6$ & $64.3 \pm 1.9$ & $67.5 \pm 2.6$ & $72.2 \pm 2.4$ \\
\hline Spinetoram & $80.1 \pm 1.5$ & $84.3 \pm 1.0$ & $86.2 \pm 1.9$ & $90.1 \pm 0.9$ & $94.3 \pm 1.7$ & $92.2 \pm 0.9$ & $77.1 \pm 1.3$ & $81.3 \pm 2.1$ & $78.2 \pm 1.7$ \\
\hline Fenpyroximate & $81.8 \pm 3.4$ & $83.3 \pm 1.5$ & $81.5 \pm 1.6$ & $85.4 \pm 1.4$ & $93.7 \pm 1.4$ & $89.9 \pm 1.5$ & $73.5 \pm 1.2$ & $80.2 \pm 1.7$ & $75.9 \pm 3.3$ \\
\hline Spirodiclofen & $80.7 \pm 0.7$ & $82.8 \pm 2.6$ & $80.4 \pm 1.1$ & $82.6 \pm 1.5$ & $86.4 \pm 0.9$ & $87.2 \pm 1.2$ & $77.9 \pm 2.5$ & $81.3 \pm 1.3$ & $74.8 \pm 1.2$ \\
\hline Difenoconazole & $86.0 \pm 0.8$ & $88.4 \pm 1.1$ & $83.2 \pm 0.7$ & $90.2 \pm 1.5$ & $94.3 \pm 0.5$ & $91.8 \pm 0.9$ & $63.2 \pm 3.9$ & $66.1 \pm 2.8$ & $62.1 \pm 2.7$ \\
\hline Azoxystrobin & $86.7 \pm 1.6$ & $89.4 \pm 1.7$ & $87.9 \pm 0.9$ & $89.3 \pm 0.5$ & $101.4 \pm 1.1$ & $95.3 \pm 1.3$ & $81.3 \pm 2.3$ & $84.7 \pm 1.5$ & $83.5 \pm 1.3$ \\
\hline Pyraclostrobin & $80.2 \pm 2.7$ & $85.2 \pm 0.5$ & $80.1 \pm 1.1$ & $84.1 \pm 1.0$ & $93.6 \pm 2.3$ & $86.7 \pm 1.0$ & $72.7 \pm 3.5$ & $82.3 \pm 1.8$ & $77.6 \pm 2.9$ \\
\hline Carbosulfan & $71.4 \pm 2.2$ & $78.3 \pm 3.5$ & $71.0 \pm 1.5$ & $75.4 \pm 3.4$ & $85.3 \pm 0.8$ & $87.0 \pm 0.7$ & $58.3 \pm 4.6$ & $73.6 \pm 3.3$ & $60.9 \pm 2.4$ \\
\hline Spirotetramat & $78.5 \pm 0.9$ & $85.1 \pm 0.7$ & $87.9 \pm 1.9$ & $92.2 \pm 2.0$ & $95.5 \pm 0.9$ & $94.3 \pm 2.4$ & $77.2 \pm 0.9$ & $73.2 \pm 1.2$ & $75.8 \pm 2.9$ \\
\hline Propargite & $78.3 \pm 1.1$ & $89.5 \pm 2.1$ & $87.1 \pm 1.0$ & $84.8 \pm 0.6$ & $104.5 \pm 0.4$ & $89.1 \pm 0.6$ & $73.5 \pm 1.5$ & $75.8 \pm 0.9$ & $71.8 \pm 3.3$ \\
\hline Pyridaben & $83.6 \pm 0.5$ & $82.1 \pm 1.2$ & $80.9 \pm 0.8$ & $87.3 \pm 1.1$ & $92.7 \pm 1.0$ & $89.7 \pm 0.2$ & $81.2 \pm 1.1$ & $81.0 \pm 2.4$ & $79.8 \pm 3.0$ \\
\hline Etoxazole & $79.5 \pm 0.7$ & $90.6 \pm 1.1$ & $89.6 \pm 0.9$ & $83.7 \pm 1.0$ & $95.6 \pm 0.7$ & $94.2 \pm 1.0$ & $76.3 \pm 4.1$ & $78.5 \pm 2.5$ & $72.4 \pm 1.7$ \\
\hline Chlorpyrifos & $82.6 \pm 1.4$ & $82.8 \pm 0.8$ & $82.9 \pm 1.4$ & $86.2 \pm 0.7$ & $93.3 \pm 1.2$ & $90.2 \pm 1.5$ & $80.8 \pm 1.7$ & $81.9 \pm 1.0$ & $79.7 \pm 3.0$ \\
\hline Thiophanate-methyl & $78.7 \pm 1.0$ & $81.9 \pm 2.3$ & $79.0 \pm 0.7$ & $84.4 \pm 2.2$ & $89.8 \pm 0.5$ & $85.7 \pm 1.0$ & $79.9 \pm 1.8$ & $77.0 \pm 3.6$ & $75.7 \pm 2.2$ \\
\hline Propiconazole & $86.7 \pm 1.7$ & $85.2 \pm 0.8$ & $85.9 \pm 1.1$ & $90.5 \pm 0.9$ & $95.7 \pm 0.8$ & $106.5 \pm 1.4$ & $73.2 \pm 0.7$ & $73.9 \pm 2.6$ & $73.7 \pm 1.4$ \\
\hline Pyrethrin & $81.2 \pm 0.5$ & $82.5 \pm 0.6$ & $82.4 \pm 1.5$ & $85.2 \pm 1.3$ & $95.3 \pm 1.3$ & $92.1 \pm 0.7$ & $58.4 \pm 5.7$ & $70.4 \pm 2.7$ & $70.5 \pm 3.3$ \\
\hline Flusilazole & $78.3 \pm 1.1$ & $79.1 \pm 1.1$ & $77.7 \pm 1.2$ & $83.6 \pm 1.1$ & $90.2 \pm 0.9$ & $88.4 \pm 0.9$ & $75.3 \pm 0.9$ & $77.1 \pm 2.9$ & $75.7 \pm 1.3$ \\
\hline Hexaconazole & $80.7 \pm 0.6$ & $82.3 \pm 1.5$ & $86.9 \pm 1.7$ & $87.4 \pm 1.4$ & $93.5 \pm 1.5$ & $93.5 \pm 2.3$ & $75.7 \pm 1.3$ & $75.4 \pm 2.5$ & $76.3 \pm 0.9$ \\
\hline Tebuconazole & $78.7 \pm 1.2$ & $78.3 \pm 3.6$ & $77.4 \pm 3.6$ & $80.8 \pm 3.1$ & $88.9 \pm 2.4$ & $86.3 \pm 1.5$ & $75.5 \pm 2.9$ & $72.6 \pm 3.4$ & $78.9 \pm 1.9$ \\
\hline Triadimefon & $81.9 \pm 1.5$ & $77.5 \pm 0.9$ & $74.6 \pm 0.7$ & $82.7 \pm 0.6$ & $87.8 \pm 0.8$ & $86.1 \pm 0.6$ & $70.2 \pm 1.5$ & $79.2 \pm 1.4$ & $72.0 \pm 2.6$ \\
\hline Thiamethoxam & $83.2 \pm 0.8$ & $81.2 \pm 1.7$ & $84.0 \pm 1.2$ & $82.7 \pm 1.7$ & $92.1 \pm 1.1$ & $90.1 \pm 1.1$ & $73.1 \pm 1.9$ & $72.9 \pm 2.6$ & $72.8 \pm 1.6$ \\
\hline Pendimethalin & $76.1 \pm 2.3$ & $84.9 \pm 1.3$ & $84.8 \pm 1.8$ & $82.8 \pm 1.3$ & $99.4 \pm 0.6$ & $93.6 \pm 1.3$ & $77.7 \pm 1.4$ & $80.3 \pm 1.7$ & $84.9 \pm 0.9$ \\
\hline Metalaxyl & $86.3 \pm 1.5$ & $86.0 \pm 0.9$ & $86.1 \pm 0.8$ & $92.1 \pm 0.9$ & $97.6 \pm 1.6$ & $94.3 \pm 0.8$ & $84.2 \pm 1.0$ & $83.5 \pm 2.1$ & $81.4 \pm 1.2$ \\
\hline Imidacloprid & $79.1 \pm 3.4$ & $87.4 \pm 1.8$ & $89.5 \pm 2.8$ & $83.2 \pm 2.5$ & $94.7 \pm 0.7$ & $95.6 \pm 1.2$ & $86.4 \pm 2.4$ & $84.6 \pm 1.6$ & $85.7 \pm 2.1$ \\
\hline Dimethoate & $72.9 \pm 1.7$ & $70.6 \pm 4.1$ & $76.9 \pm 0.9$ & $79.6 \pm 1.2$ & $81.3 \pm 1.3$ & $82.2 \pm 0.5$ & $67.4 \pm 3.5$ & $68.5 \pm 2.3$ & $69.4 \pm 3.4$ \\
\hline Acetamiprid & $84.7 \pm 0.6$ & $84.7 \pm 1.2$ & $83.8 \pm 2.2$ & $87.8 \pm 0.9$ & $97.4 \pm 0.5$ & $90.4 \pm 1.4$ & $78.4 \pm 2.5$ & $83.2 \pm 1.8$ & $81.3 \pm 1.5$ \\
\hline 3-hydroxyl Carbofuran & $84.3 \pm 0.9$ & $85.2 \pm 2.8$ & $83.4 \pm 0.6$ & $83.8 \pm 2.9$ & $95.7 \pm 2.0$ & $94.1 \pm 2.1$ & $81.8 \pm 1.0$ & $81.7 \pm 0.7$ & $77.7 \pm 1.5$ \\
\hline Pymetrozine & $79.9 \pm 1.8$ & $85.1 \pm 0.9$ & $85.7 \pm 0.8$ & $89.9 \pm 1.0$ & $94.6 \pm 0.8$ & $97.2 \pm 0.9$ & $74.5 \pm 2.7$ & $73.3 \pm 1.9$ & $76.1 \pm 1.7$ \\
\hline Omethoate & $76.5 \pm 2.1$ & $83.0 \pm 0.7$ & $84.4 \pm 2.3$ & $80.5 \pm 1.3$ & $90.3 \pm 1.7$ & $91.2 \pm 1.7$ & $75.3 \pm 1.1$ & $80.1 \pm 0.8$ & $79.2 \pm 2.4$ \\
\hline Carbendazim & $82.3 \pm 1.4$ & $88.8 \pm 1.4$ & $87.0 \pm 0.5$ & $96.3 \pm 1.4$ & $97.8 \pm 0.9$ & $96.0 \pm 2.9$ & $89.7 \pm 2.3$ & $88.6 \pm 2.8$ & $83.9 \pm 0.9$ \\
\hline Methomyl & $83.5 \pm 0.8$ & $83.3 \pm 0.8$ & $82.2 \pm 1.0$ & $95.7 \pm 0.7$ & $93.9 \pm 0.6$ & $89.3 \pm 2.0$ & $70.3 \pm 1.9$ & $71.5 \pm 1.3$ & $73.7 \pm 1.0$ \\
\hline
\end{tabular}

A: $200 \mathrm{mg} \mathrm{C18+200} \mathrm{mg} \mathrm{PSA+15} \mathrm{mg} \mathrm{GCB;} \mathrm{B:} 200 \mathrm{mg}$ C18+200 mg PSA+45 mg GCB; C: $200 \mathrm{mg}$ C18+200 mg PSA+90 mg GCB; D: 400mg C18+400 mg PSA+15 mg GCB; E: $400 \mathrm{mg} \mathrm{C} 18+400 \mathrm{mg}$ PSA+45 mg GCB; F: $400 \mathrm{mg} \mathrm{C18+400} \mathrm{mg} \mathrm{PSA+90} \mathrm{mg} \mathrm{GCB;} \mathrm{G:} 600 \mathrm{mg}$ C18+600 mg PSA+25 mg GCB; H: 600mg C18+600 mg PSA+45 mg GCB; I: $600 \mathrm{mg}$ C18+600 mg PSA+90 mg GCB. SD: standard deviation 
those obtained with the $200 \mathrm{mg}$ adsorbents (C18 and PSA) and the $600 \mathrm{mg}$ adsorbents (C18 and PSA). However, GCB strongly adsorbed some pesticides leading to low recoveries of these analytes (less than 70\%). Therefore, $400 \mathrm{mg} \mathrm{C18,} 400 \mathrm{mg}$ PSA and $45 \mathrm{mg}$ GCB was selected for purification in this experiment.

\subsection{Method validation}

\section{Matrix effects}

It has been reported that matrix effects (ME) are common when analysing pesticide residues by LC-MS/MS. ME are caused by the influence of co-eluting compounds on the ionization efficiency of the electrospray interface in the LC-MS/MS analysis, and the effects manifest as ion enhancement or inhibition (Chawla et al., 2017; Galani et al., 2018). The wolfberry is rich in mineral substances, proteins, polysaccharose, amino acids, carotinoid, flavonoids (Gong et al., 2018). In this work, the matrix effect was calculated by the following Equation 7:

$M E=A_{\text {Matrix }} / A_{S}$
Where $\mathrm{A}_{\text {Matrix }}$ is the peak area of matrix standard sample and $\mathrm{A}_{s}$ is the peak area of pure solvent standard sample.

The ME of 31 pesticide residues were determined, and the ME values were split into three groups based (0.8-1.2, higher than 1.2 and less than 0.8 ). ME values between 0.8 and 1.2 were classified as low ME, which can be ignored; $\mathrm{ME}$ values higher than 1.2 were deemed matrix enhancements; and ME values less than 0.8 were classified as matrix suppression (Fan et al., 2013). As shown in Table 2, the ME values of 28 pesticide residues were between 0.8 and 1.2, and could be ignored. The ME values of 2 pesticide residues were less than 0.8 , indicating matrix suppression. The ME values of carbendazim were higher than 1.2 , indicating matrix enhancement. The above experimental results showed that although the extractant solution was purified, some interfering substances that inhibited the analysis of the target analytes remained in the solution. This phenomenon was consistent with the results found by other researchers (Prodhan et al., 2016). To compensate for the matrix inhibition effects, a matrix standard curve was used to quantify 31 pesticide residues in the wolfberry samples.

Table 2 Performance characteristics of the optimized method

\begin{tabular}{|c|c|c|c|c|c|c|}
\hline Components & $\mathrm{ME}(\%)$ & Liner range $(\mu \mathrm{g} / \mathrm{L})$ & Linear equation & $\mathrm{R}^{2}$ & LODs $(\mu \mathrm{g} / \mathrm{kg})$ & LOQs $(\mu \mathrm{g} / \mathrm{kg})$ \\
\hline Abamectin & 0.86 & $1 \sim 100$ & $y=10918 x-589$ & 0.9991 & 0.5 & 1 \\
\hline Spinetoram & 1.04 & $1 \sim 100$ & $y=97035 x+4682$ & 0.9986 & 0.5 & 1 \\
\hline Fenpyroximate & 0.84 & $1 \sim 100$ & $y=21453 x+7294$ & 0.9967 & 0.5 & 1 \\
\hline Spirodiclofen & 0.82 & $1 \sim 100$ & $y=17456 x-1384$ & 0.9957 & 0.5 & 1 \\
\hline Difenoconazole & 0.95 & $1 \sim 100$ & $y=74291 x-8792$ & 0.9978 & 0.5 & 1 \\
\hline Azoxystrobin & 0.88 & $1 \sim 100$ & $y=59640 x+2843$ & 0.9956 & 0.5 & 1 \\
\hline Pyraclostrobin & 0.89 & $1 \sim 100$ & $y=89751 x-3927$ & 0.9969 & 0.5 & 1 \\
\hline Carbosulfan & 0.84 & $1 \sim 100$ & $y=113544 x+9390$ & 0.9976 & 0.5 & 1 \\
\hline Spirotetramat & 0.91 & $1 \sim 100$ & $y=42842 x+8253$ & 0.9988 & 0.5 & 1 \\
\hline Propargite & 0.68 & $1 \sim 100$ & $y=60124 x+5764$ & 0.9987 & 0.5 & 1 \\
\hline Pyridaben & 0.74 & $1 \sim 100$ & $y=109223 x+9823$ & 0.9986 & 0.5 & 1 \\
\hline Etoxazole & 0.79 & $1 \sim 100$ & $y=25389 x-1946$ & 0.9988 & 0.5 & 1 \\
\hline Chlorpyrifos & 1.01 & $1 \sim 100$ & $y=30321 x-2043$ & 0.9987 & 0.5 & 1 \\
\hline Thiophanate-methyl & 0.77 & $1 \sim 100$ & $y=94742 x+3809$ & 0.9988 & 0.5 & 1 \\
\hline Propiconazole & 0.89 & $1 \sim 100$ & $y=173024 x-17645$ & 0.9958 & 0.5 & 1 \\
\hline Pyrethrin & 0.74 & $1 \sim 100$ & $y=87356 x+10362$ & 0.9979 & 0.5 & 1 \\
\hline Flusilazole & 0.93 & $1 \sim 100$ & $y=21443 x+1974$ & 0.9979 & 0.5 & 1 \\
\hline Hexaconazole & 0.86 & $1 \sim 100$ & $y=123154 x+6532$ & 0.9978 & 0.5 & 1 \\
\hline Tebuconazole & 0.88 & $1 \sim 100$ & $y=51837 x+4071$ & 0.9958 & 0.5 & 1 \\
\hline Triadimefon & 0.92 & $1 \sim 100$ & $y=89374 x-7163$ & 0.9968 & 0.5 & 1 \\
\hline Thiamethoxam & 1.06 & $1 \sim 100$ & $y=12584 x-1487$ & 0.9986 & 0.5 & 1 \\
\hline Pendimethalin & 0.83 & $1 \sim 100$ & $y=151656 x-16158$ & 0.9957 & 0.5 & 1 \\
\hline Metalaxyl & 0.98 & $1 \sim 100$ & $y=73625 x+3729$ & 0.9988 & 0.5 & 1 \\
\hline Imidacloprid & 0.87 & $1 \sim 100$ & $y=123722 x-8645$ & 0.9989 & 0.5 & 1 \\
\hline Dimethoate & 0.89 & $1 \sim 100$ & $y=185697 x+6719$ & 0.9957 & 0.5 & 1 \\
\hline Acetamiprid & 0.95 & $1 \sim 100$ & $y=129413 x+30723$ & 0.9989 & 0.5 & 1 \\
\hline 3-hydroxyl Carbofuran & 1.09 & $1 \sim 100$ & $y=98735 x-9547$ & 0.9988 & 0.5 & 1 \\
\hline Pymetrozine & 0.91 & $1 \sim 100$ & $y=60124 x+5764$ & 0.9986 & 0.5 & 1 \\
\hline Omethoate & 0.76 & $1 \sim 100$ & $y=49485 x+9897$ & 0.9979 & 0.5 & 1 \\
\hline Carbendazim & 1.48 & $1 \sim 100$ & $y=60747 x+3085$ & 0.9984 & 0.5 & 1 \\
\hline Methomyl & 0.65 & $1 \sim 100$ & $y=83519 x+2785$ & 0.9994 & 0.5 & 1 \\
\hline
\end{tabular}

ME: matrix effect; $\mathrm{R}^{2}$ : linear correlation coefficient; LOD: limit of detection; LOQ: Limit of Quantification. 


\section{Selectivity}

The selectivity of the detection method is important for the qualitative and quantitative analysis of 31 pesticide residues because the blank matrix solution did not contain parent ions and fragment ions of the target analytes at a detectable level. Based on a comparison of the chromatograms of the matrixmatched standard solution and the blank matrix solution (Figure 2), after QuEChERS purification, the peaks of residual co-extracting compounds did not interfere, as they appeared at different retention times, confirming the excellent selectivity of the established method.

\section{Linearity and detectability of the method}

In the linearity studies, all the standard working solutions were determined under the optimal chromatography and mass spectrometry conditions. Linear regression analysis was performed on a plot with concentration on the X-axis, and the peak area on the Y-axis. The results shown in Table 2 indicate that suitable linearities were obtained in the corresponding concentration range of each pesticide residue, and the coefficients of determination ( $\mathrm{R}^{2}$ values) were higher than 0.99 .

The LODs and LOQs of the method were calculated according to the validated experimental results. The results showed that the LODs and LOQs of this method the LODs were $0.5 \mu \mathrm{g} / \mathrm{kg}$ and $1.0 \mu \mathrm{g} / \mathrm{kg}$, respectively (Table 2 ), which are consistent with those of the methods reported for pesticide residue analysis in cereals (Bordin et al., 2016), vegetables (Xiu-Ping et al., 2017), fruits (Stachniuk et al., 2017), juices (Rizzetti, et al., 2016) and other foods (Kasiotis et al., 2014).

\section{Accuracy and precision of the method}

The accuracy and precision of the method were assessed for each pesticide residue by determining the recoveries and the RSDs from blank wolfberry samples spiked at three different levels. The results are shown in Table 3. The average recoveries were in the range of $73.8 \%-111.5 \%$, and the RSDs were less than $10 \%$. Thus, the accuracy and precision of the 31 pesticide residues in wolfberry are acceptable.

\subsection{Actual sample ananlysis and risk assessment}

Pesticide residues in wolfberry

Among the 200 wolfberry samples from Northwest China, 23 pesticides were detected and samples containing pesticide residues were in $83.5 \%$ of the wolfberry samples. The levels of individual pesticides in the samples are shown in Table 4. The detection rate exceeded $5 \%$ for of 14 pesticides and ranged from $7.5-79 \%$ for all 23 pesticides, with acetamiprid, carbendazim and imidacloprid being detected in 79\%, 50\% and $43 \%$ of the samples, respectively. In these samples, the residues of 10 pesticides exceed the maximum residue limit (MRL) in GB 2763-2019 (China, 2019) for wolfberry. Among them, pyridaben, acetamiprid and difenoconazole exceeded the MRL the most frequently, exceeding the MRL in $19.5 \%, 12 \%$ and $11 \%$ of the wolfberry samples, respectively. In this work, it was found that abamectin, pymetrozine, spinetoram, etoxazole, omethoate, dimethoate, triadimenol, chlorpyrifos, pendimethalin and fenpyroximate were detected in wolfberry samples for the first time, and the detection rates of etoxazole, triadimenol, and pendimethalin were more than $10 \%$. The amounts and types of pesticide residues in wolfberry differed in different regions, but acetamiprid, imidacloprid carbendazim, pyridaben, propargite and thiamethoxam were detected in samples from all regions.
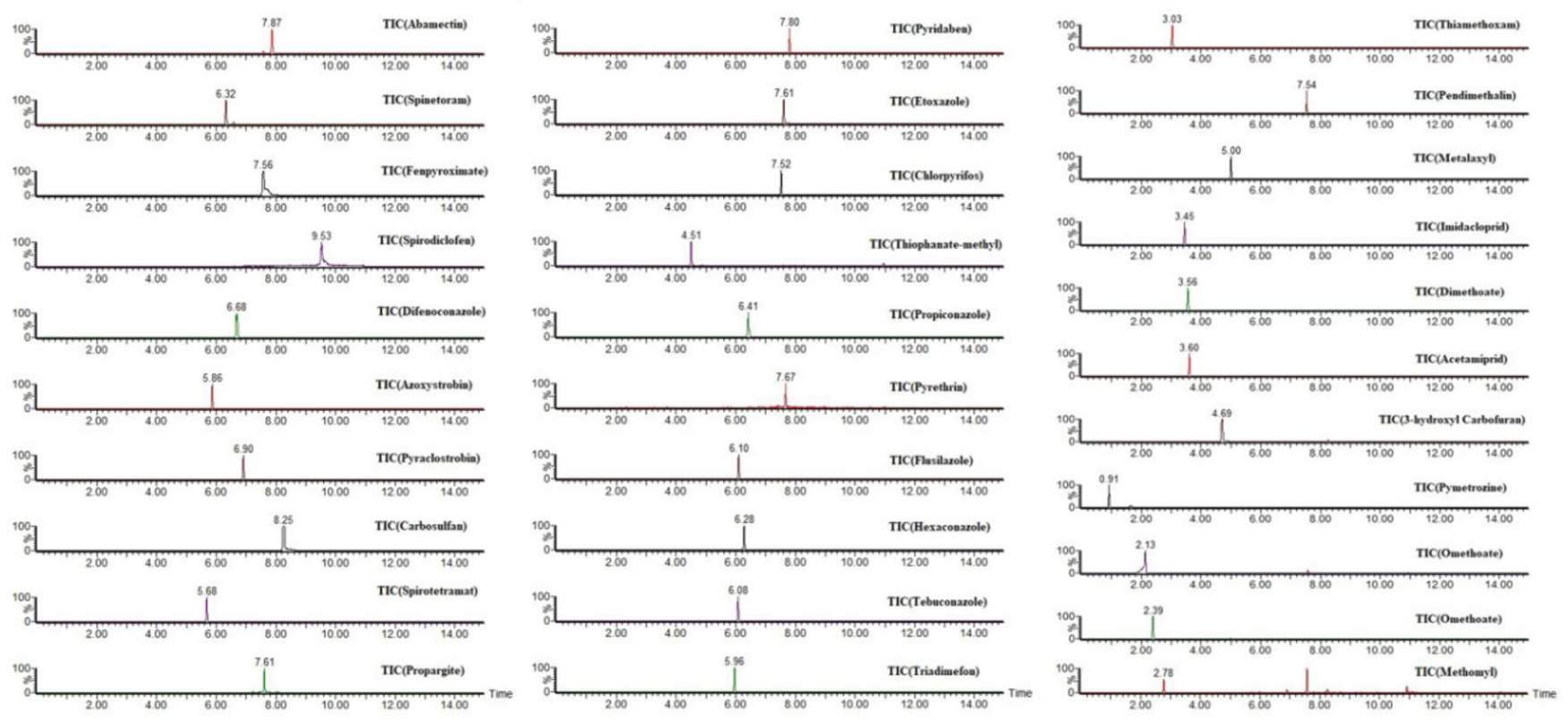

Figure 2. TIC of LC-MS/MS containing the 31 pesticides (Spiked $20 \mu \mathrm{g} / \mathrm{L}$ ). 
Table 3. Average recovery and RSD of 31 pesticides in the wolfberry $(n=6)$.

\begin{tabular}{|c|c|c|c|c|c|c|}
\hline \multirow[b]{2}{*}{ Components } & \multicolumn{2}{|c|}{$0.01 \mathrm{mg} / \mathrm{kg}$} & \multicolumn{2}{|c|}{$0.02 \mathrm{mg} / \mathrm{kg}$} & \multicolumn{2}{|c|}{$0.1 \mathrm{mg} / \mathrm{kg}$} \\
\hline & $\begin{array}{c}\text { Average } \\
\text { recovery }(\%)\end{array}$ & RSD (\%) & $\begin{array}{c}\text { Average } \\
\text { recovery (\%) }\end{array}$ & RSD (\%) & $\begin{array}{c}\text { Average } \\
\text { recovery (\%) }\end{array}$ & RSD (\%) \\
\hline Abamectin & 71.2 & 2.9 & 78.9 & 4.1 & 79.2 & 1.9 \\
\hline Spinetoram & 94.8 & 6.9 & 91.5 & 4.7 & 111.7 & 2.5 \\
\hline Fenpyroximate & 86.9 & 2.2 & 82.3 & 2.5 & 91.7 & 2.3 \\
\hline Spirodiclofen & 82.4 & 3.8 & 84.2 & 3.6 & 87.4 & 4.5 \\
\hline Difenoconazole & 87.3 & 5.5 & 99.1 & 2.1 & 100.4 & 1.1 \\
\hline Azoxystrobin & 90.2 & 2.7 & 89.7 & 4.4 & 93.6 & 5.9 \\
\hline Pyraclostrobin & 95.6 & 3.6 & 89.5 & 1.1 & 96.3 & 2.5 \\
\hline Carbosulfan & 85.5 & 2.1 & 87.7 & 4.0 & 88.3 & 1.1 \\
\hline Spirotetramat & 85.1 & 6.2 & 83.2 & 2.9 & 93.2 & 3.5 \\
\hline Propargite & 80.5 & 3.1 & 81.5 & 2.0 & 84.9 & 3.1 \\
\hline Pyridaben & 90.0 & 6.2 & 88.9 & 2.3 & 92.1 & 3.8 \\
\hline Etoxazole & 89.5 & 4.9 & 85.1 & 2.3 & 86.2 & 4.5 \\
\hline Chlorpyrifos & 91.8 & 6.4 & 90.2 & 2.9 & 95.8 & 2.6 \\
\hline Thiophanate-methyl & 86.5 & 2.6 & 95.7 & 4.8 & 97.6 & 2.1 \\
\hline Propiconazole & 86.2 & 5.0 & 91.1 & 4.9 & 94.2 & 3.4 \\
\hline Pyrethrin & 95.4 & 4.6 & 82.4 & 1.7 & 85.1 & 2.9 \\
\hline Flusilazole & 80.0 & 5.0 & 82.3 & 2.5 & 92.1 & 1.9 \\
\hline Hexaconazole & 107.2 & 1.2 & 98.0 & 2.3 & 104.2 & 2.7 \\
\hline Tebuconazole & 87.4 & 5.4 & 93.2 & 3.7 & 92.3 & 2.3 \\
\hline Triadimefon & 89.3 & 3.9 & 94.2 & 2.8 & 96.4 & 2.6 \\
\hline Thiamethoxam & 90.2 & 2.9 & 96.1 & 3.4 & 98.7 & 1.2 \\
\hline Pendimethalin & 82.6 & 4.5 & 87.9 & 4.1 & 91.8 & 2.7 \\
\hline Metalaxyl & 84.8 & 4.0 & 85.3 & 4.9 & 93.7 & 3.1 \\
\hline Imidacloprid & 90.6 & 3.3 & 100.3 & 6.0 & 100.0 & 4.1 \\
\hline Dimethoate & 84.9 & 2.7 & 88.4 & 3.9 & 89.9 & 4.1 \\
\hline Acetamiprid & 84.1 & 4.0 & 91.4 & 4.2 & 92.6 & 3.8 \\
\hline 3-hydroxyl Carbofuran & 90.3 & 2.8 & 95.4 & 1.9 & 100.3 & 2.7 \\
\hline Pymetrozine & 96.4 & 4.0 & 90.2 & 3.5 & 97.5 & 1.6 \\
\hline Omethoate & 85.1 & 4.5 & 91.5 & 3.7 & 95.3 & 1.7 \\
\hline Carbendazim & 93.0 & 1.3 & 98.6 & 2.0 & 109.7 & 3.9 \\
\hline Methomyl & 89.1 & 3.7 & 89.5 & 1.5 & 96.6 & 3.5 \\
\hline
\end{tabular}

RSD: relative standard deviation.

On the one hand, wolfberry is susceptible to pests during its growth, and correspondingly, more and more pesticides will be used. On the other hand, overuse of pesticide has resulted in excessive pesticide residues.

\section{Risk posed by chronic dietary intake of pesticide residues in wolfberry}

The risk of the chronic dietary intake of the 23 detected pesticide residues was calculated. As shown in Table 5, the chronic dietary intake risk (\%ADI) was much less than $100 \%$, ranging from $0.0001 \%$ to $1.6067 \%$, with an average of $0.1254 \%$. The $\% A D I$ of omethoate was slightly higher than $1 \%$, at $1.6067 \%$, while the $\% A D I$ of difenoconazole, acetamiprid, dimethoate and 3 -hydroxyl carbofuran ranged from $0.10 \%$ to $0.60 \%$. The $\% A D I$ values of the other 18 pesticides were below $0.1 \%$. These results showed that the risk posed by chronic dietary intake of the pesticide residues in wolfberry produced in Northwest China is acceptable. The $H Q$ for the cumulative chronic dietary risk assessment of wolfberry was 0.02569 , which is much less than 1 and indicates that the cumulative risk posed by chronic dietary intake is also acceptable.

\section{Risk posed by acute dietary intake of pesticide residues in} wolfberry

According to the World Health Organization (WHO) database (Philippe et al., 2020), the ARfD data for thiophanate methyl, spinetoram, propargite, thiophanate-methyl and azoxystrobin are "inconclusive", and there are no $A R f D$ data for avermectin, pyridaben, any bactericide and omethoate. The $A R f D$ values for the other 10 pesticides are shown in Table 6. As shown in Table 6, the risk posed by acute dietary intake of these 10 pesticides is much less than $100 \%$, ranging from $0.0010 \%$ to $0.4999 \%$, with an average of $0.26001 \%$. These results showed that the risk posed by acute dietary intake of the pesticide residues in 
Table 4. Residue levels of 23 pesticides in wolfberry.

\begin{tabular}{|c|c|c|c|c|}
\hline Pesticide & \% Positive & Range (mg/kg) & MRL(mg/kg) & \%ex MRL \\
\hline Avermectin & 1.0 & $0.0243 \sim 0.0289$ & 0.01 & $1.0 \%$ \\
\hline Difenoconazole & 20.0 & $0.0011 \sim 0.3200$ & 0.01 & $11.0 \%$ \\
\hline Imidacloprid & 43.0 & $0.00222 \sim 1.4118$ & 1.00 & $2.0 \%$ \\
\hline Pymetrozine & 0.5 & 0.0757 & - & - \\
\hline Pyridaben & 33.0 & $0.00187 \sim 0.5601$ & 0.01 & $19.5 \%$ \\
\hline Acetamiprid & 79.0 & $0.00168 \sim 5.7647$ & 2.00 & $12.0 \%$ \\
\hline Carbendazim & 50.0 & $0.0008 \sim 1.9272$ & 1.00 & $3.5 \%$ \\
\hline Spinetoram & 2.0 & $0.00624 \sim 0.0184$ & - & - \\
\hline Thiophanate-Methyl & 0.5 & 0.0154 & - & - \\
\hline Spirodiclofen & 20.5 & $0.0033 \sim 1.0459$ & - & - \\
\hline Propargite & 25.5 & $0.0015 \sim 4.0889$ & - & - \\
\hline Tebuconazole & 25.0 & $0.0013 \sim 2.2664$ & - & - \\
\hline Etoxazole & 15.5 & $0.0009 \sim 0.4672$ & - & - \\
\hline Omethoate & 2.0 & $0.0040 \sim 0.3103$ & 0.01 & $1.5 \%$ \\
\hline Thiamethoxam & 13.0 & $0.0017 \sim 2.1038$ & - & - \\
\hline Dimethoate & 3.5 & $0.0014 \sim 0.0297$ & - & - \\
\hline 3-hydroxyl Carbofuran & 7.5 & $0.0010 \sim 0.0594$ & 0.01 & $2.0 \%$ \\
\hline Azoxystrobin & 3.0 & $0.0012 \sim 0.0435$ & - & - \\
\hline Triadimefon & 4.0 & $0.0025 \sim 0.0900$ & 1.00 & $0.0 \%$ \\
\hline Triadimenol & 18.0 & $0.0025 \sim 2.0588$ & - & - \\
\hline Chlorpyrifos & 7.5 & $0.0015 \sim 0.6741$ & 0.10 & $1.5 \%$ \\
\hline Pendimethalin & 10.0 & $0.0018 \sim 0.0178$ & - & - \\
\hline Fenpyroximate & 3.0 & $0.0022 \sim 0.0600$ & 0.50 & $0.0 \%$ \\
\hline
\end{tabular}

MRL: maximum residue limit.

Table 5. \%ADI, HQ and $H I$ of pesticide residues in wolfberry.

\begin{tabular}{|c|c|c|c|c|c|}
\hline Pesticide & STMR $(\mathrm{mg} / \mathrm{kg})$ & $A D I(\mathrm{mg} / \mathrm{kg})$ & $A D I \%$ & $H Q$ & $H I$ \\
\hline Avermectin & 0.0266 & 0.001 & 0.5320 & 0.00474 & \\
\hline Difenoconazole & 0.0642 & 0.01 & 0.0360 & 0.00032 & \\
\hline Imidacloprid & 0.2733 & 0.06 & 0.0480 & 0.00043 & \\
\hline Pymetrozine & 0.0757 & 0.03 & 0.0505 & 0.00045 & \\
\hline Pyridaben & 0.0465 & 0.01 & 0.0296 & 0.00026 & \\
\hline Acetamiprid & 0.8091 & 0.07 & 0.1031 & 0.00092 & \\
\hline Carbendazim & 0.2027 & 0.03 & 0.0114 & 0.00010 & \\
\hline Spinetoram & 0.0121 & 0.02 & 0.0119 & 0.00011 & \\
\hline Thiophanate-Methyl & 0.0154 & 0.09 & 0.0034 & 0.00003 & \\
\hline Spirodiclofen & 0.1371 & 0.01 & 0.0593 & 0.00053 & \\
\hline Propargite & 0.3854 & 0.01 & 0.0237 & 0.00021 & \\
\hline Tebuconazole & 0.19 & 0.03 & 0.0154 & 0.00014 & \\
\hline Etoxazole & 0.0428 & 0.05 & 0.0038 & 0.00003 & \\
\hline Omethoate & 0.0906 & 0.0003 & 1.6067 & 0.01432 & \\
\hline Thiamethoxam & 0.1258 & 0.08 & 0.0024 & 0.00002 & \\
\hline Dimethoate & 0.0137 & 0.002 & 0.1030 & 0.00092 & \\
\hline 3-hydroxyl Carbofuran & 0.0129 & 0.001 & 0.1333 & 0.00119 & \\
\hline Azoxystrobin & 0.0084 & 0.2 & 0.0001 & 0.00000 & \\
\hline Triadimefon & 0.0327 & 0.03 & 0.0133 & 0.00012 & \\
\hline Triadimenol & 0.2129 & 0.03 & 0.0371 & 0.00033 & \\
\hline Chlorpyrifos & 0.0804 & 0.01 & 0.0326 & 0.00029 & \\
\hline Pendimethalin & 0.0054 & 0.1 & 0.0009 & 0.00001 & \\
\hline \multirow[t]{2}{*}{ Fenpyroximate } & 0.0209 & 0.01 & 0.0246 & 0.00022 & \\
\hline & & & & & 0.02569 \\
\hline
\end{tabular}

STMR: supervised trial median residue; $A D I$ : acceptable daily intake; \%ADI: acceptable daily intake percentage; $H Q$ : hazard quotient; $H I$ : hazard index. 
Table 6. \%ARfD, SM, HQ and $H I$ of pesticide residues in wolfberry.

\begin{tabular}{|c|c|c|c|c|c|c|}
\hline Pesticide & $H R(\mathrm{mg} / \mathrm{kg})$ & $A R f D(\mathrm{mg} / \mathrm{kg})$ & ARfD\% & $S M(\mathrm{mg} / \mathrm{kg})$ & $H Q$ & $H I$ \\
\hline Avermectin & 0.0289 & - & - & - & & \\
\hline Difenoconazole & 0.3200 & 0.3 & 0.3005 & 106.5 & 0.0026787 & \\
\hline Imidacloprid & 1.4118 & 0.4 & 0.3999 & 353.0 & 0.0035654 & \\
\hline Pymetrozine & 0.0757 & 0.1 & - & - & & \\
\hline Pyridaben & 0.5601 & - & - & - & & \\
\hline Acetamiprid & 5.7647 & 0.1 & - & - & & \\
\hline Carbendazim & 1.9272 & 0.1 & 0.4999 & 385.5 & 0.0044569 & \\
\hline Spinetoram & 0.0184 & - & - & - & & \\
\hline $\begin{array}{l}\text { Thiophanate- } \\
\text { Methyl }\end{array}$ & 0.0154 & Unnecessary & - & - & & \\
\hline Spirodiclofen & 1.0459 & Unnecessary & - & - & & \\
\hline Propargite & 4.0889 & Unnecessary & - & - & & \\
\hline Tebuconazole & 2.2664 & 0.3 & - & - & & \\
\hline Etoxazole & 0.4672 & Unnecessary & - & - & & \\
\hline Omethoate & 0.3103 & - & - & - & & \\
\hline Thiamethoxam & 2.1038 & 1 & - & - & & \\
\hline Dimethoate & 0.0297 & 0.02 & 0.0200 & 148.5 & 0.0001784 & \\
\hline $\begin{array}{l}\text { 3-hydroxyl } \\
\text { Carbofuran }\end{array}$ & 0.0594 & 0.001 & 0.0010 & 5950.0 & 0.0000089 & \\
\hline Azoxystrobin & 0.0435 & Unnecessary & - & - & & \\
\hline Triadimefon & 0.0900 & 0.08 & 0.0800 & 112.5 & 0.0007132 & \\
\hline Triadimenol & 2.0588 & 0.08 & 0.0800 & 2575.0 & 0.0007128 & \\
\hline Chlorpyrifos & 0.6741 & 0.1 & 0.999 & 675.0 & 0.0008903 & \\
\hline Pendimethalin & 0.0178 & 1 & 0.1998 & 8.9 & 0.0017808 & \\
\hline \multirow[t]{2}{*}{ Fenpyroximate } & 0.0600 & 0.02 & 0.0200 & 300.0 & 0.0001783 & \\
\hline & & & & & & 0.015164 \\
\hline
\end{tabular}

HR: highest residue; $A R f D$ : acute reference dose; \%ARfD: acute reference dose percentage; SM: safety margin; HQ: hazard quotient; $H I$ : hazard index.

wolfberry from Northwest China is acceptable and very low. The HQ for the cumulative acute dietary risk assessment of wolfberry is 0.015164 , which is far less than 1 and indicates that the cumulative acute dietary risk is also acceptable. As shown in Table 6, the maximum concentration of each pesticide was far less than the MRL, which confirms that the risk posed by acute dietary intake of these pesticides is very low.

\section{Conclusions}

A modified QuEChERS method coupled with LC-MS/MS was established and validated for the determination of 31 pesticides residues in wolfberry. A sorbent of $400 \mathrm{mg} \mathrm{C18,} 400 \mathrm{mg}$ PSA and $45 \mathrm{mg}$ GCB was used as the dSPE sorbent for sample cleanup; 200 wolfberry samples were analysed; and 23 pesticides were detected. The most frequently detected pesticides were carbendazim, pyridaben, propargite, thiamethoxam, acetamiprid and imidacloprid. These results were compared with pesticide residues detected in samples from the other three main wolfberry production areas. The pesticides detected in different areas differed, although some pesticides were common to all regions. This finding illustrated that a greater number of pesticides are present in wolfberry from Northwest China, and the increase in pesticide residues in wolfberry necessitate continuous monitoring to ensure the safety of wolfberry consumption. The chronic dietary intake, acute dietary intake and cumulative risk of 23 pesticides in wolfberry were estimated for adults, and the results showed that the exposure to pesticides was quite low. The results of the risk assessment in this study demonstrated that the pesticide residues in wolfberry do not represent a potential risk to human health.

\section{Conflict of interest}

The authors declare no conflicts of interest.

\section{Funding}

This research was funded by Key Programs for Science and Technology Development of Xinjiang Production and Construction Corps, China., grant number No2018AB011 and Major Project of National Agricultural Product Quality and Safety Risk Assessment, grant number GJFP2019020.

\section{Author contributions}

Conceptualization, Lijie Xing and Yuan Wang; sample collection, Ruifeng Luo and Xianyi Li; sample analysis, Liangjun Zou and Yuan Wang; statistical analysis, Lijie Xing and Ruifeng Luo; writing-original draft preparation, Lijie Xing and Yuan Wang; writing-review and editing, Yuan Wang. All authors have read and agreed to the published version of the manuscript. 


\section{Acknowledgements}

The authors wish to thank the anonymous reviewers, whose insightful comments and helpful suggestions significantly contributed to improving this paper.

\section{References}

Ali, M. C., Chen, J., Zhang, H., Li, Z., Zhao, L., \& Qiu, H. (2019). Effective extraction of flavonoids from Lycium barbarum L. fruits by deep eutectic solvents-based ultrasound-assisted extraction. Talanta, 203, 16-22. http://dx.doi.org/10.1016/j.talanta.2019.05.012. PMid:31202321.

Amagase, H., \& Farnsworth, N. R. (2011). A review of botanical characteristics, phytochemistry, clinical relevance in efficacy and safety of Lycium barbarum fruit (Goji). Food Research International, 44(7), 1702-1717. http://dx.doi.org/10.1016/j.foodres.2011.03.027.

Anastassiades, M., Lehotay, S. J., Štajnbaher, D., \& Schenck, F. J. (2003). Fast and easy multiresidue method employing acetonitrile extraction/partitioning and "dispersive solid-phase extraction" for the determination of pesticide residues in produce. Journal of AOAC International, 86(2), 412-431. http://dx.doi.org/10.1093/ jaoac/86.2.412. PMid:12723926.

Bordin, A. B., Minetto, L., do Nascimento Filho, I., Beal, L. L., \& Moura, S. (2016). Determination of pesticide residues in whole wheat flour using modified QuEChERS and LC-MS/MS. Food Analytical Methods, 10(1), 1-9. http://dx.doi.org/10.1007/s12161-016-0542-2.

Chawla, S., Patel, H. K., Gor, H. N., Vaghela, K. M., Solanki, P. P., \& Shah, P. G. (2017). Evaluation of matrix effects in multiresidue analysis of pesticide residues in vegetables and spices by LC-MS/ MS. Journal of AOAC International, 100(3), 616-623. http://dx.doi. org/10.5740/jaoacint.17-0048. PMid:28300025.

Chen, J.-N., Lian, Y.-J., Zhou, Y.-R., Wang, M.-H., Zhang, X.-Q., Wang, J.-H., Wu, Y.-N., \& Wang, M.-L. (2019). Determination of 107 Pesticide Residues in Wolfberry with Acetate-buffered Salt Extraction and Sin-QuEChERS Nano Column Purification Coupled with Ultra Performance Liquid Chromatography Tandem Mass Spectrometry. Molecules (Basel, Switzerland), 24(16), 2918-2941. http://dx.doi. org/10.3390/molecules24162918. PMid:31408943.

China. Ministry of Agriculture and Rural Affairs of the People's Republic of China. China's National Health Commission. State Administration for Market Regulation. (2019). National food safety standard: Maximum residue limits forpesticides in food (GB 27632019). Beijing: Standards Press of China. (In chinese)

Chiu, K., Zhou, Y., Yeung, S.-C., Lok, C. K.-M., Chan, O. O.-C., Chang, R. C.-C., So, K.-F., \& Chiu, J.-F. (2010). Up-regulation of crystallins is involved in the neuroprotective effect of wolfberry on survival of retinal ganglion cells in rat ocular hypertension model. Journal of Cellular Biochemistry, 110(2), 311-320. http://dx.doi.org/10.1002/ jcb.22539. PMid:20336662.

Donno, D., Mellano, M. G., Raimondo, E., Cerutti, A. K., Prgomet, Z., \& Beccaro, G. L. (2016). Influence of applied drying methods on phytochemical composition in fresh and dried goji fruits by HPLC fingerprint. European Food Research and Technology, 242(11), 19611974. http://dx.doi.org/10.1007/s00217-016-2695-z.

Fan, S., Zhao, P., Yu, C., Pan, C., \& Li, X. (2013). Simultaneous determination of 36 pesticide residues in spinach and cauliflower by LC-MS/MS using multi-walled carbon nanotubes-based dispersive solid-phase clean-up. Food Additives \& Contaminants. Part A, Chemistry, Analysis, Control, Exposure \& Risk Assessment, 31(1), 73-82. http://dx.doi.or g/10.1080/19440049.2013.853324. PMid:24350649.
Fu, Y., Yang, T., Zhao, J., Zhang, L., Chen, R., \& Wu, Y. (2017). Determination of eight pesticides in Lycium barbarum by LC-MS/ MS and dietary risk assessment. Food Chemistry, 218, 192-198. http://dx.doi.org/10.1016/j.foodchem.2016.09.014. PMid:27719897.

Galani, J. H., Houbraken, M., Wumbei, A., Djeugap, J., Fotio, D., \& Spanoghe, P. (2018). Evaluation of 99 pesticide residues in major agricultural products from the western highlands zone of cameroon using QuEChERS method extraction and LC-MS/MS and GCECD analyses. Foods, 7(11), 184-201. http://dx.doi.org/10.3390/ foods7110184. PMid:30405079.

Gan, L., Hua Zhang, S., Liang Yang, X., \& Bi Xu, H. (2004). Immunomodulation and antitumor activity by a polysaccharide-protein complex from Lycium barbarum. International Immunopharmacology, 4(4), 563 569. http://dx.doi.org/10.1016/j.intimp.2004.01.023. PMid:15099534.

Gong, G., Dang, T., Deng, Y., Han, J., Zou, Z., Jing, S., Zhang, Y., Liu, Q., Huang, L., \& Wang, Z. (2018). Physicochemical properties and biological activities of polysaccharides from Lycium barbarum prepared by fractional precipitation. International Journal of Biological Macromolecules, 109, 611-618. http://dx.doi.org/10.1016/j. ijbiomac.2017.12.017. PMid:29222018.

Huang, X., Xue, J., Wang, Y., Wu, X., \& Tong, H. (2012). Rapid simultaneous determination of organochlorine and pyrethroid pesticide residues in Lycium barbarum L. using gas chromatography with electron-capture detector. Analytical Methods, 4(4), 1132-1141. http://dx.doi.org/10.1039/c2ay05625a.

Kasiotis, K. M., Anagnostopoulos, C., Anastasiadou, P., \& Machera, K. (2014). Pesticide residues in honeybees, honey and bee pollen by LC-MS/MS screening: reported death incidents in honeybees. The Science of the Total Environment, 485-486, 633-642. http://dx.doi. org/10.1016/j.scitotenv.2014.03.042. PMid:24747255.

Li, W., Shen, S., Chen, H., \& Guo, Q. (2020). Dissipation study and dietary risk assessment of dinotefuran, DN, and UF in wolfberry. International Journal of Environmental Analytical Chemistry, 100(13), 1524-1535. http://dx.doi.org/10.1080/03067319.2019.1655557.

Liu, J., Ali, A., Yu, M., Zhu, F., \& Kidane, D. (2015). Risk evaluation of main pests and integrated management in chinese wolfberry, Lycium barbarum L. Pakistan Journal of Zoology, 47(1), 21-29.

Lu, W., Jiang, Q., Shi, H., Niu, Y., Gao, B., \& Yu, L. (2014). Partial LeastSquares-Discriminant Analysis Differentiating Chinese Wolfberries by UPLC-MS and Flow Injection Mass Spectrometric (FIMS) Fingerprints. Journal of Agricultural and Food Chemistry, 62(37), 9073-9080. http://dx.doi.org/10.1021/jf502156n. PMid:25152955.

Luo, Q., Cai, Y., Yan, J., Sun, M., \& Corke, H. (2004). Hypoglycemic and hypolipidemic effects and antioxidant activity of fruit extracts from Lycium barbarum. Life Sciences, 76(2), 137-149. http://dx.doi. org/10.1016/j.lfs.2004.04.056. PMid:15519360.

Oshita, D., \& Jardim, I. C. S. F. (2014). Comparison of different sorbents in the QuEChERS method for the determination of pesticide residues in strawberries by LC-MS/MS. Chromatographia, 77(19-20), 12911298. http://dx.doi.org/10.1007/s10337-014-2726-5.

Philippe, V., Neveen, A., Marwa, A., \& Basel, A. A. (2020). Occurrence of pesticide residues in fruits and vegetables for the Eastern Mediterranean Region and potential impact on public health. Food Control, 119,107457. http://doi.org/10.1016/j.foodcont.2020.107457.

Potterat, O. (2010). Goji (Lycium barbarumand L. Chinense): phytochemistry, pharmacology and safety in the perspective of traditional uses and recent popularity. Planta Medica, 76(1), 7-19. http://dx.doi.org/10.1055/s-0029-1186218. PMid:19844860.

Prodhan, M. D. H., Papadakis, E.-N., \& Papadopoulou-Mourkidou, E. (2016). Analysis of pesticide residues and their variability in cabbage using QuEChERS extraction in combination with LC-MS/MS. Food 
Analytical Methods, 9(12), 3470-3478. http://dx.doi.org/10.1007/ s12161-016-0537-z.

Qian, J. (2004). The efficiency of flavonoids in polar extracts of Lycium chinense Mill fruits as free radical scavenger. Food Chemistry, 87(2), 283-288. http://dx.doi.org/10.1016/j.foodchem.2003.11.008.

Qin, X., Luo, X., Han, J., Chen, Y., Zhang, K., \& Hu, D. (2020). Residual determination of pyrethrins in Lycium barbarum (goji) by GC-MS/ MS and a dietary risk assessment of Chinese goji consumption. Food Additives \& Contaminants: Part A, 37(3), 478-487. http://dx.doi.or g/10.1080/19440049.2019.1707295.

Rizzetti, T. M., Kemmerich, M., Martins, M. L., Prestes, O. D., Adaime, M. B., \& Zanella, R. (2016). Optimization of a QuEChERS based method by means of central composite design for pesticide multiresidue determination in orange juice by UHPLC-MS/MS. Food Chemistry, 196, 25-33. http://dx.doi.org/10.1016/j.foodchem.2015.09.010. PMid:26593461.

Stachniuk, A., Szmagara, A., Czeczko, R., \& Fornal, E. (2017). LC-MS/ MS determination of pesticide residues in fruits and vegetables. Journal of Environmental Science and Health. Part. B, Pesticides, Food Contaminants, and Agricultural Wastes, 52(7), 446-457. http://dx.doi. org/10.1080/03601234.2017.1301755. PMid:28353394.
Wang, C. C., Chang, S. C., Inbaraj, B. S., \& Chen, B. H. (2010). Isolation of carotenoids, flavonoids and polysaccharides from Lycium barbarum L. and evaluation of antioxidant activity. Food Chemistry, 120(1), 184-192. http://dx.doi.org/10.1016/j.foodchem.2009.10.005.

Xiu-Ping, Z., Lin, M., Lan-Qi, H., Jian-Bo, C., \& Li, Z. (2017). The optimization and establishment of QuEChERS-UPLC-MS/MS method for simultaneously detecting various kinds of pesticides residues in fruits and vegetables. Journal of Chromatography. B, Analytical Technologies in the Biomedical and Life Sciences, 1060, 281-290. http://dx.doi.org/10.1016/j.jchromb.2017.06.008. PMid:28649028.

Zhao, J., Li, H., Xi, W., An, W., Niu, L., Cao, Y., Wang, H., Wang, Y., \& Yin, Y. (2015). Changes in sugars and organic acids in wolfberry (Lycium barbarum L.) fruit during development and maturation. Food Chemistry, 173, 718-724. http://dx.doi.org/10.1016/j. foodchem.2014.10.082. PMid:25466081.

Zhou, Z.-Q., Xiao, J., Fan, H.-X., Yu, Y., He, R.-R., Feng, X.-L., Kurihara, H., So, K.-F., Yao, X.-S., \& Gao, H. (2017). Polyphenols from wolfberry and their bioactivities. Food Chemistry, 214, 644-654. http://dx.doi. org/10.1016/j.foodchem.2016.07.105. PMid:27507521. 